\title{
Centrifugal compressor impeller loading factor analysis
}

\author{
$Y$. Galerkin ${ }^{1}, A$. Drozdov $^{1, *}$, and $A$. Rekstin $^{1}$ \\ ${ }^{1}$ Peter the Great St. Petersburg Polytechnic University, Saint Petersburg, Russia
}

\begin{abstract}
The loading factor performance modelling is an important part of centrifugal compressor performance calculation. The presented information on model stages' test data confirms the fact that the loading factor versus flow coefficient at an impeller exit is a linear function independent of Mach number (subsonic flow). The test data and the design characteristics of the series of 10 model stages are compared with the calculation of an inviscid flow and with calculations done using the NUMECA software. Math models offered by the authors, and inviscid calculations solve the problem of a primary design. The CFDcalculation for final solution is non-satisfactory. If the loading factor is calculated by total temperature difference and flow coefficient is calculated by a continuity equation, the performance is not quite linear and lies much higher. For the considered stages CFD-calculation inaccuracy is $+(0,06 \ldots 0,12)$. CFDcalculated flow coefficient is inside $0,96 \ldots 0,98$ of the measured and of the calculated by the Math model.
\end{abstract}

\section{Introduction}

The total power of centrifugal compressors in the industry of the Russian Federation exceeds $60 \mathrm{MW}$. Therefore, gas-dynamic design of centrifugal compressors is an important and remains a difficult task.

Modern design of new compressors is based on previously tested model stage' characteristics, or is executed by field-type design methods and CFDcalculations. The development of mathematical models for design methods is carried out by specialists from universities and specialized enterprises [1 - 6].

Mathematical models are solving two problems: a direct problem - calculation of gas dynamic characteristics of the centrifugal compressor stage, and an inverse problem - designing of the stage with given parameters. To solve these problems, it is necessary to calculate the mechanical work fed to the gas and calculate its part, which is spent to overcome resistance of a flow path [7].

The authors use their own Universal modeling method [7 - 10]. It is successfully used for calculation and design of industrial centrifugal compressors.

The results of CFD-calculations depend on the quality of the computational grid, the turbulence model, the calculation statement, and still are not reliable [11 14]. However, CFD calculations are widely used to calculate the gas-dynamic characteristics of centrifugal compressor stages.

To calculate a pressure rate characteristic of a centrifugal compressor, stage the efficiency and work input coefficient are necessary:

$$
\pi^{*}=\left[1+(k-1) \psi_{i} M_{u}^{2}\right]^{\frac{k}{k-1}} \eta^{*}
$$

where $\pi^{*}$ is total pressure ratio; $k$ is isentropic coefficient; $\psi_{i}$ is work coefficient; $\eta^{*}$ is total efficiency; $M_{u}$ is relative Mach number.

The work input coefficient for a closed industrial compressor is:

$$
\psi_{i}=\psi_{T}\left(1+\beta_{\text {leak }}+\beta_{f r}\right)
$$

where $\psi_{T}$ is loading factor; $\beta_{\text {leac }}$ is labyrinth seal leakage coefficient; $\beta_{f r}$ is disc friction coefficient.

Parasitic losses coefficients $\beta_{\text {leak }}$ and $\beta_{f r}$ can be calculated by some or other semi-empirical formulae with acceptable accuracy. The main problem is to calculate correctly the loading factor $\psi_{T}=c_{u 2} \cdot u_{2}$ ( $c_{u 2}$ is tangential component of absolute velocity; $u_{2}$ is impeller velocity) that in Russia is the Euler work coefficient.

The author of [16] presented the work input analysis based on a slip factor. It is ratio of circumferential velocity component $c_{u 2}$ to this component $c_{u 2 \infty}$ in case of infinite number of blades:

$$
\mu=\frac{c_{u 2}}{c_{u 2 \infty}}
$$

Here:

$\psi_{T}=c_{u 2} / u_{2}=1-\varphi_{2} c t g \beta_{2}$

\footnotetext{
*Corresponding author: a_drozdi@mail.ru
} 


$$
\psi_{T \infty}=c_{u 2 \infty} / u_{2}=1-\varphi_{2} \operatorname{ctg} \beta_{b l 2}
$$

where $\varphi_{2}$ is flow rate coefficient at impeller outlet; $\beta_{b l 2}$ is outlet blade angle; $\beta_{2}$ is flow angle at impeller outlet related to tangential direction.

The author of [16] compared slip factor formulae presented in $[17,18]$. His conclusion is that slip factor by A. Stodola [18] is closer to test data than others:

$$
\mu=1-\frac{\frac{\pi}{z} \sin \beta_{b l 2}}{1-\varphi_{2} \operatorname{ctg} \beta_{b l 2}}
$$

A. Stodola formula following from (6) for a loading factor was recommended in the book [19] and it is still popular in Russian engineering society

$$
\psi_{T}=1-\varphi_{2} \operatorname{ctg} \beta_{b l 2}-\frac{\pi}{z} \sin \beta_{b l 2}
$$

In accordance with (7), the characteristic $\psi_{T}=f\left(\varphi_{2}\right)$ is a straight line parallel to the characteristic $\psi_{T \infty}=1-\varphi_{2} \operatorname{ctg} \beta_{b l 2}$ of the impeller with infinite number of blades. The loading factor of the impeller with infinite number of blades at zero flow rate is $\psi_{T \infty 0}=1$. Its characteristic line $\psi_{T \infty}=f\left(\varphi_{2}\right)$ is inclined to the ordinate axis with the angle that is equal to the exit blade angle: $\beta_{T}=\beta_{b l 2}$. In accordance with Eq. (7) the characteristic line of the real impeller $\psi_{T}=f\left(\varphi_{2}\right)$ is also a straight line inclined to the ordinate axis with the angle that is equal to the exit blade angle: $\beta_{T}=\beta_{b l 2}$.

If being correct, the Eq. (7) resolves the problem of the loading factor characteristic simulation. However, the design experience of authors has demonstrated that Eq. (7) is more or less correct for $2 \mathrm{D}$ impellers with arc blade mean line and angles $\beta_{b l 2} \approx 45^{0}$ only. The reliable simulation of $\psi_{T}=f\left(\varphi_{2}\right)$ by simple algebraic formula is not resolved still and hardly can be resolved by simple formula. The aim of this contribution is to demonstrate typical empirical loading factor characteristics and what can be done to their simulation by inviscid flow calculations and CFD.

\section{Methods}

Research is done using the following tools: quasi-threedimensional calculations and hydro-gas-dynamic program packages NUMECA Fine/Turbo. The Q-3-D inviscid flow calculation 3DM.023 program is the combination of quasi-orthogonal solution for axissymmetric flow, and integral equations solution (blades are substitutes by vortexes) for blade-to blade surfaces. NUMECA Fine/Turbo, based, like other popular CFD programs, on the Reynolds averaged system of Navier-Stokes equations. The results of numerical simulation were compared with experimental data.

\subsection{Objects under study}

Series of model stages of industrial centrifugal compressors were developed and tested in the Laboratory of compressor problem [21]. To obtain data for $\psi_{T}=f\left(\varphi_{2}\right)$ the measured parameters are reduced.

Work coefficient calculated by measured increase of total temperature:

$$
\psi_{i}=\frac{c_{p} \Delta T^{*}}{u_{2}^{2}}
$$

where $T^{*}$ is total temperature; $c_{p}$ is isobaric heat capacity.

Loading factor in accordance with Eq. (2):

$$
\psi_{T}=\frac{\psi_{i}}{1+\beta_{\text {leak }}+\beta_{f r}}
$$

Coefficients of parasitic or losses are calculated by semi-empirical equations ( $K_{f r}$ and $K_{\text {leak }}$ are empirical coefficients of the Math model):

$$
\begin{aligned}
& \beta_{f r}=K_{f r} \frac{\varepsilon_{2}}{\Phi \times \psi_{T} \times \operatorname{Re}_{u}^{0,2}} \\
& \beta_{\text {leak }}=K_{\text {leak }} \frac{K_{D} \bar{\delta}_{\text {seal }}}{\Phi \times \bar{D}_{1}}
\end{aligned}
$$

where $\Phi$ is flow rate coefficient; $\bar{D}_{1}$ is relative impeller inlet diameter; $\varepsilon_{2}$ is densities' ratio at exit of impeller and at an impeller inlet; $K_{D}$ is geometry parameter of $2 \mathrm{D}$ impeller; $\mathrm{Re}_{u}$ is impeller diameter Reynolds number

$\mathrm{K}$. Soldatova in [15] has demonstrated that in order to increase the accuracy of impeller characteristics modeling it is necessary to take into account the shroud disk labyrinth seal leakage flow. The flow rate of the impeller is bigger than the flow rate of the stage due to leakage flow: $\bar{m}_{0}=\bar{m}+\bar{m}_{\text {leack }}$ ( $\bar{m}$ is mass flow rate).

Measured flow rate of an impeller is represented by the flow rate coefficient:

$$
\Phi=\frac{\bar{m}}{\frac{\pi}{4} D_{2}^{2} u_{2} \rho_{i n l}^{*}}
$$

where $\rho_{i n l}^{*}$ is total inlet gas density.

The impeller flow rate coefficient is: 


$$
\Phi_{0}=\Phi\left(1+\beta_{\text {leack }}\right)
$$

$$
\text { As } \quad \bar{m}_{0}=\Phi_{0} \frac{\pi}{4} D_{2}^{2} u_{2} \rho_{\text {inl }}^{*}=\varphi_{20} \pi D_{2} b_{2} \rho_{2} \quad\left(b_{2} \quad\right. \text { is }
$$

impeller outlet width of channel) a flow rate coefficient at the impeller exit is:

$$
\varphi_{20}=\frac{\Phi_{0}}{4 \varepsilon_{2} \bar{b}_{2}}
$$

Compressibility factor $\varepsilon_{2}=\rho_{2} / \rho_{0}^{*}$ calculation is based on the known thermodynamic equations with polytrophic exponent being a function of polytrophic efficiency $\frac{n}{n-1}=\eta \frac{k}{k-1}$. The principles and specific features of the efficiency math model are presented in [10].

\section{Results}

\subsection{Samples of empirical characteristics and an inviscid flow calculation}

Names of the model stages contain information about design parameters. As the sample below we present the model stage 028/058-0373 ( $\Phi_{\text {des }}=0.28, \psi_{T \text { des }}=0.58$, $\bar{D}_{h}=0.373$ ) from the family $20 \mathrm{CE}$. The stage consists of the impeller with cylindrical blades, vaneless diffuser and return channel.

The range $\Phi=0.020-0.040$ is typical for regimes of this stage as a part of a compressor. The math modeling in this range is accurate enough. The information on flow parameters at three points of characteristic is presented in the table 1.

The flow rate coefficient, by which stage parameters were measured, is presented in column 1. The work coefficient and polytrophic efficiency at full parameters calculated using measured flow parameters are shown in columns 6 and 7. The values of parameters calculated with mathematical model based on test results are presented in columns $2,4,5$. By flow rate coefficients in column 3 Q-3-D inviscid flow is calculated with $M_{u}=$ 0,70 . It is shown in [22] that inviscid velocity diagrams are close to measured velocity diagrams at design flow rate.

The area of real diagrams in general decreases by 5 $10 \%$ as viscosity diminishes circulation. The values of loading factor for inviscid flow program are shown in column 10, and the ratio of experimental and inviscid loading factor is in column 11. According to obvious equation for the straight line, the values in columns 4 and 10 define inclination angle of the loading factor characteristic, which is equal to $\beta_{T i d}=29.7^{\circ}$, and loading factor is $\psi_{T 0 \text { id }}=0.891$. This agrees accurately with the inclination angle of tested impeller with blunt trailing edge - Fig. 3 below. By infinite number of blades the inclination angle is equal to blade exit angle ( $\beta_{b l 2}=30,30$ for impeller 028/058-0373).

By infinite number of blades loading factor for zero flow rate equals to one. For the impeller 028/058-0373 by the inviscid gas flow this loading factor is equal to 0,891 , i.e. the finite number of blades diminishes coefficient by $10.9 \%$. For real impeller type 028 this coefficient equals 0.835 . I.e. the viscosity diminishes the loading factor coefficient for zero flow rate further by $5.6 \%$.

Empirical loading factor measured points of the impeller 028/058-0373 and approximation straight line on Fig. 1 give impression about the function $\psi_{T}=f\left(\varphi_{2}\right)$ configuration and test accuracy.

In all known cases, the function $\psi_{T}=f\left(\varphi_{2}\right)$ is a straight line. According to [22], the function $\psi_{T}=f\left(\varphi_{2}\right)$ is linear and does not depend on Mach number if the flow is subsonic. Dimensional work input characteristics of multistage compressors are practically linear, too. Factory tests prove that in [15].

The linearity of loading factor characteristic of an ideal impeller with infinite number of blades is evident. The linear connection of a loading factor with a flow rate coefficient is a fact and could be used for analysis and calculation.

The impeller 028/058-0373 was tested with three different configurations of blade trailing edge. Blunt and

Table 1. Parameters of the stage type 028 and its loading factor characteristic by $M_{u}=0.70$.

\begin{tabular}{|l|l|l|l|l|l|l|l|l|l|l|}
\hline $\mathbf{1}$ & $\mathbf{2}$ & $\mathbf{3}$ & $\mathbf{4}$ & $\mathbf{5}$ & $\mathbf{6}$ & $\mathbf{7}$ & $\mathbf{8}$ & $\mathbf{9}$ & $\mathbf{1 0}$ & $\mathbf{1 1}$ \\
\hline$\Phi$ & $\beta_{\text {leak }}$ & $\Phi_{0}$ & $\varphi_{20}$ & $\beta_{f r}$ & $\psi_{i}$ & $\psi_{T}$ & $\eta^{*}$ & $n$ & $\psi_{T \text { id }}$ & $\frac{\psi_{T}}{\psi_{\text {Tid }}}$ \\
\hline 0.0375 & 0.0151 & 0.0381 & 0.188 & 0.0316 & 0.537 & $\begin{array}{l}0.513 \\
(0,572)\end{array}$ & 0.768 & 1.592 & 0.561 & 0.914 \\
\hline 0.0325 & 0.0174 & 0.0331 & 0.160 & 0.0338 & 0.588 & $\begin{array}{l}0.559 \\
(0,620)\end{array}$ & 0.800 & 1.556 & 0.609 & 0.918 \\
\hline 0.0219 & 0.0258 & 0.0225 & 0.107 & 0.0432 & 0.653 & $\begin{array}{l}0.647 \\
(0,711)\end{array}$ & 0.755 & 1.609 & 0.703 & 0.920 \\
\hline
\end{tabular}




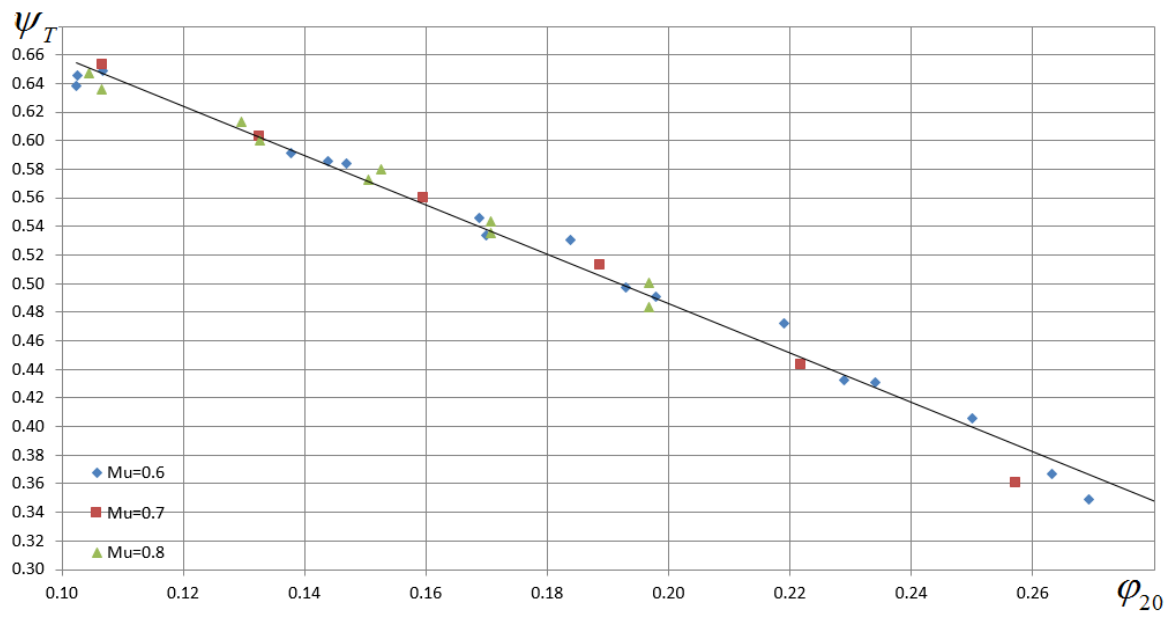

Fig. 1. Loading factor of the impeller $028 / 058-0373$ at $M_{u}=0,60,0,70,0,80$.

tampered from the front side trailing edges are shown on Fig. 1. In [7] tapering with the front or back side is recommended for "tuning" the loading factor. It is possible to increase (tapering with the back surface) or decrease (tapering with the front surface) the loading factor.

The impeller 028/058-0373 was tested with blunt trailing edge, with tapering $100 \%$ as it is shown on Fig. 1 and with a partial tapering: "tapering 50\%". The trailing edge in this case is $50 \%$ blunt. Fig. 2 demonstrates comparison of three empirical characteristics with calculated characteristics.
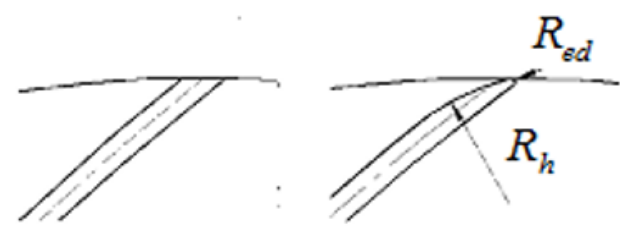

Fig. 2. Two configurations of impeller's blade trailing edge. Left - blunt t.e. Right - tapering 100\% of the front side.

The experimental points presented in Fig. 3 are approximated by straight lines.

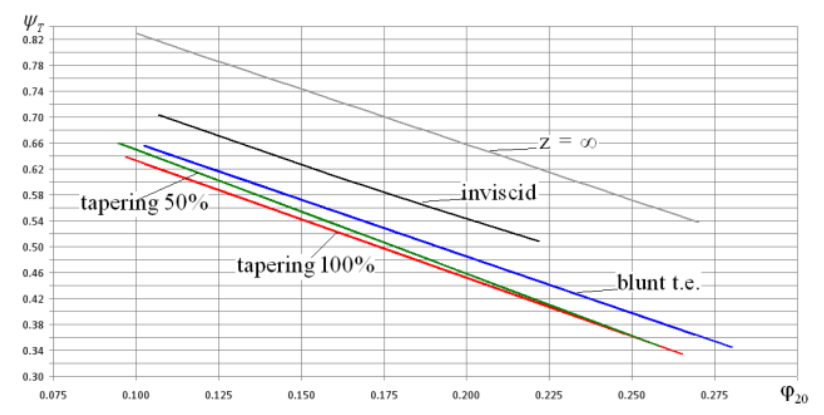

Fig. 3. Loading factor characteristics of the impeller 028/0580373 .

It is remarkable that the tampering influences the characteristic irregularly. By 50\% tampering the value of loading factor at zero flow rate is practically the same, as by blunt trailing edge $\left(\psi_{T 0}\right.$ is equal 0.835 and 0,836 respectively). Nevertheless, because of smaller inclination angle $\beta_{T}$, the design flow rate is smaller. Inclination angle $\beta_{T}$ at $100 \%$ tampering is practically the same as at blunt trailing edge.

The fact, that experimental characteristics are practically parallel to characteristic for infinite number of blades is, unfortunately, not universal. Otherwise, the modeling of $\psi_{T}=f\left(\varphi_{2}\right)$ would be much more easy. By big exit angles, the angle $\beta_{T}$ is substantially smaller than blades' exit angle

\subsection{CFD-simulation of loading factor performance}

To explore the modeling potential of CFD-methods, the loading factor characteristic of the impeller 028/0580373 were calculated with NUMECA Fine Turbo program. The calculations were performed for one blade channel. Parasitic losses were not simulated. Turbulence model Spallart-Almarras was used. The number of grid elements was chosen according to recommendations formulated in [10].

In Fig. 4 the measured characteristic $\psi_{T}=f\left(\varphi_{2}\right)$ are compared with calculated loading factor characteristics. Alike measured, the calculated characteristics at different $\mathrm{M}_{\mathrm{u}}$ are similar in the most important range of flow rates. In certain flow rate range calculated characteristic is practically linear. But main result is that calculated loading factor is bigger by $7-9 \%$. This error is not acceptable in design practice.

The same result is presented in other publications $[23,24]$. The reason why $\psi_{\mathrm{T} \text { calk }}>\psi_{\mathrm{T}}$ may be connected with different values of flow coefficient $\varphi_{20}$. The function $\varphi_{2}=\mathrm{f}(\Phi)$ based on test data is presented on Fig. 5 by the red line. Shown on Fig. 5 flow rate coefficient was calculated using NUMECA Fine Turbo program in two ways. 


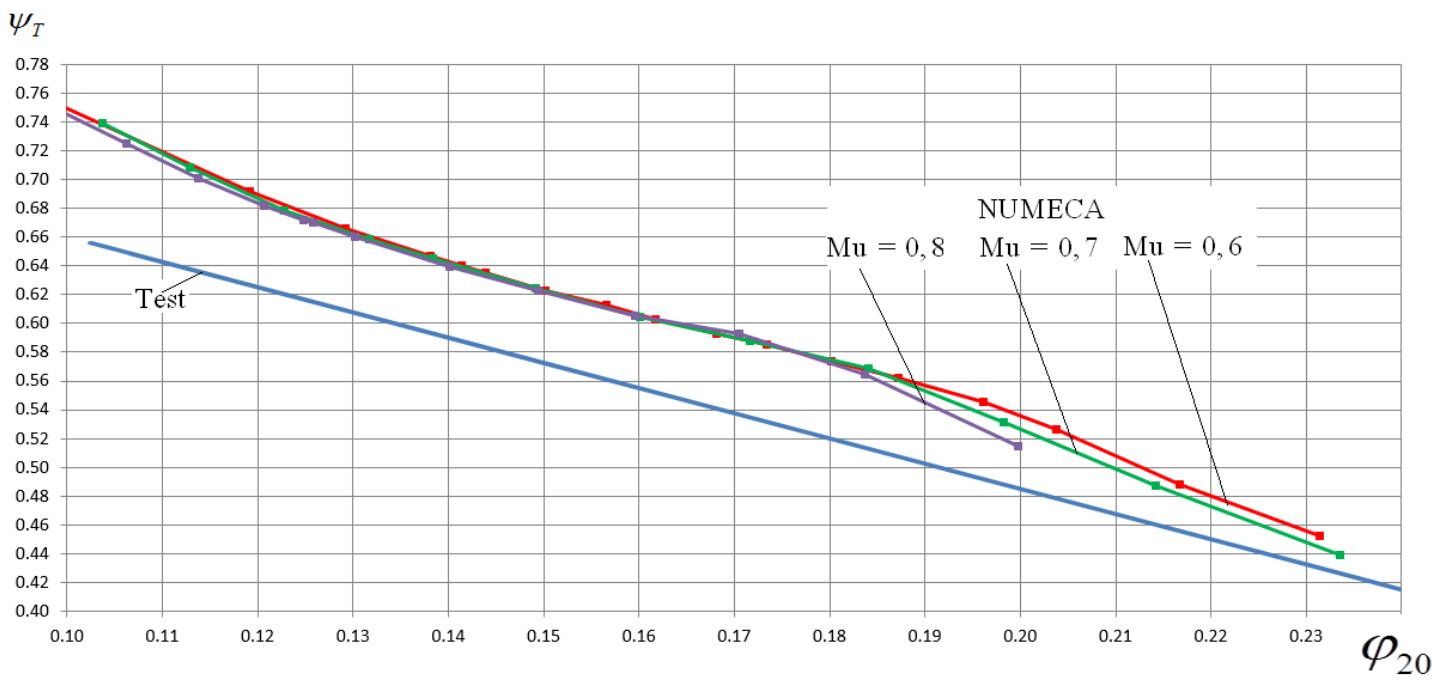

Fig. 4. Loading factor of the impeller 028/058-0373 at $M_{u}=0,60,0,70,0,80$.

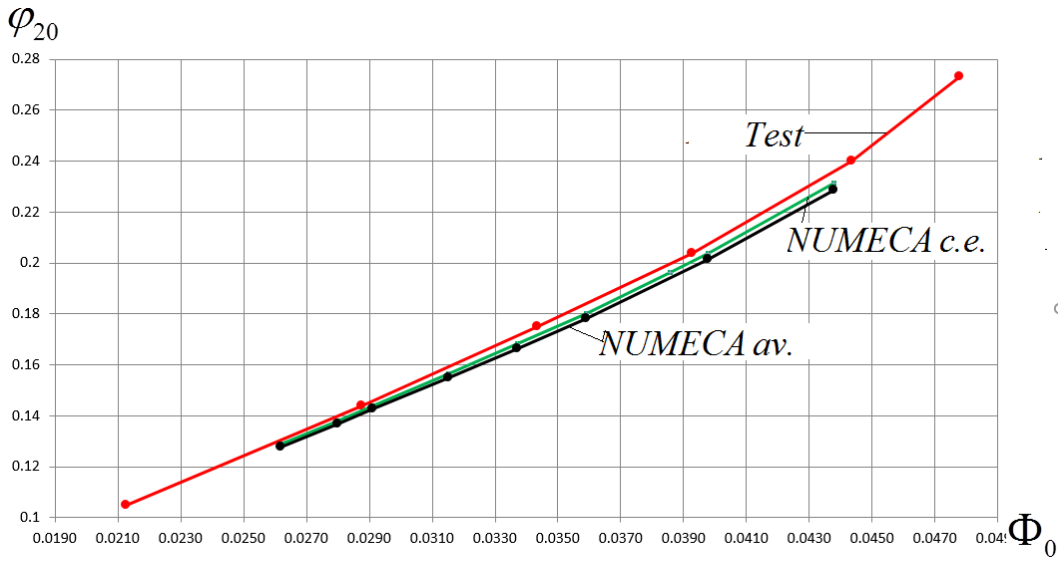

Fig. 5. Flow coefficient at the impeller exit $\varphi_{20}$ versus flow rate coefficient $\Phi_{0}$.

First - according to continuity equation:

$\varphi_{20}=\frac{\bar{m}_{0}}{\rho_{2} \pi D_{2} b_{2} u_{2}}$

Second - averaged value at the impeller exit:

$\mathrm{c}_{\mathrm{r} 2 \mathrm{av}}=\frac{\sum_{\mathrm{s}} \mathrm{c}_{\mathrm{r} 2} \cdot|\mathrm{d} \mathrm{s}|}{\sum_{\mathrm{s}}\left|\mathrm{d}^{\mathrm{r}}\right|}$

Two ways calculated NUMECA flow rate coefficients differ little. Continuity equation gives result that is slightly close to the test. The smaller flow coefficient leads to higher loading factor. At $\Phi_{0}=0,033$ the calculated $\varphi_{20}$ is smaller by $2 \%$. It increases the calculated loading factor by $1,5 \%$. But it is only one part of the problem. The authors of [24] compare results of calculations of the whole impeller and one blade channel, different turbulence models, "Stage" and "Frozen rotor" interface, steady and unsteady flow, etc.
No satisfactory simulation of the loading factor characteristic was obtained.

\section{Conclusions}

Correct simulation of loading factor characteristic is necessary for successful design. Simple semi-empirical equations as A. Stodola one and alike cannot provide correct solution. These equations do not take into account all details of an impeller flow path.

The authors' design experience has shown that Q-3$\mathrm{D}$ inviscid flow calculated loading factor performance is close to a real characteristic and reflects all details of an impeller flow path. Some empirical correlations lead to correct calculation of $\psi_{\mathrm{T}}=\mathrm{f}\left(\varphi_{2}\right)$, which solves the problem on the engineering level.

The calculations were performed using the supercomputer center SCC "Polytechnic". The research was supported by a grant of Russian Science Foundation (project No. 18-7910165). 


\section{References}

[1] D. Japikse, E.M. Krivitzky, Proceedings of the ASME Turbo Expo DOI: 10.1115/GT2016-57956 (2016)

[2] D. Japikse, JSME fluid engineering conference. $34-44$ (2003)

[3] D. Japikse JANNAF Conference. (Cleveland. Ohio) (1998)

[4] V.E. Evdokimov, The bank of experimental data on model levels and their elements for the design of MCC. Turbines and compressors. 2, 3-4 (1997)

[5] A.V. Semakov, V.E. Evdokimov, A.I. Reprintsev, A.N. Lyubimov, Modernization of centrifugal compressors on nitrogen production. Compressors and pneumatics. 5, 20-22 (2013)

[6] A.T. Lunev, Development of highly efficient replaceable flow parts of centrifugal compressors for gas pumping units. Dis ... Cand. tech. sciences. 123 (2005)

[7] Y. Galerkin, Turbocompressors. LTD information and publishing center KHT. Moscow. 596 (2010)

[8] Y.B. Galerkin, K. Danilov, E. Popova, Inernational Conference on Compressors and their Systems. ( London: City University, UK.) 465-480 (1999)

[9] Y.B. Galerkin, Communications of the Union of German Engineers. 947 (Germany, Hannover) (1992)

[10] A.A. Drozdov, Design method of centrifugal compressors with 3D impellers. PhD thesis. 236 (2016)

[11] R. Matas, T. Syka, L. Hurda, EPJ Web of Conferences

180, https://doi.org/10.1051/epjconf/201818002060 EFM 2017 (2018)

[12] S.C.Kowalski, J.E. Pacheco, S. Fakhri, J.M. Sorokes, Proc. of the Forty-FirstTurbomachinery Symposium 24-31 (2012)

[13] W. Kryłłowicz, P. Świder, Z. Kozanecki, K. Kabalyk, J.Z. Kozanecki, 12th European Conf. on Turbomachinery. 45 - 52 (2017)

[14] P. Le Sausse, P. Fabrie, D. Arnou, F. Clunet, EPJ Web of Conferences. 45 DOI: 10.1051/epjconf/20134501059 (2013)

[15] K.V. Soldatova, The creation of new mathematical models of the centrifugal compressors flow part and a database of model stages. Dr. of tech. Sci thesis 257 (2017)

[16] F.J. Wiesner, Trans ASME Journal of Engineering for Power 89, 58 - 72 (1967)

[17] J.D. Stanitz, Trans ASME 72, 473 - 97 (1952)

[18] A. Stodola, Steam and Gas turbines. 1356

[19] V.F. Ries, Centrifugal compressor machines. (Leningrad, Machinostroenie) 351 (1981)

[20] A.N. Karpov, A method of loading factor characteristics of the centrifugal compressorimpeller according to the results of model stages tests. PhD thesis 137 (2011)

[21] Y.B. Galerkin, Proceedings of the scientific school of compressor engineering SPbGPU. (SPb, "SPbSPU") 670 (2010)
[22] K.P. Seleznev, Y.B. Galerkin Centrifugal compressors. (Leningrad, Machinostroenie) 271 (1982)

[23] D.M. Gamburger Numerical modeling of viscous gas flow in a centrifugal compressor stage:methods and results PhD thesis. 190 (2009)

[24] A.I. Borovkov, I.B. Voinov, Y.B. Galerkin, A.G. Nikiforov, M.A. Nikitin Modeling of gas-dynamic characteristics on the example of a model stage of a centrifugal compressor. Scientific and Technical Bulletin of SPbPU. Natural and engineering sciences. 24, 2 4457. DOI: 10.18721 / JEST.24.2018.4 (2018) 